\title{
A Surface Plasmon Resonance Bio-Sensor based on Dual Core D-Shaped Photonic Crystal Fibre Embedded with Silver Nanowires for Multi-Sensing
}

\author{
Suoda Chu, K. Nakkeeran, Senior Member, IEEE, Abdosllam M. Abobaker, Member, IEEE, Sumeet S. Aphale, \\ Senior Member, IEEE, S. Sivabalan, P. Ramesh Babu and K. Senthilnathan
}

\begin{abstract}
In this paper, for sensing and monitoring the biochemical analyte dissolved in liquid, antigen-antibody interaction or protein-DNA/RNA binding process, we design a surface plasmon resonance refractive index based biosensor by using a dual core D-shaped six-fold photonic crystal fibre which is embedded with silver nanowires for multi-detection. We numerically analyze both the dispersion relations and the loss spectra for various analytes by finite element method. This optical fibre bio-sensor monitors the changes of the refractive index for different analytes by measuring the spectral shifts of the fibre loss peaks at their resonance wavelengths. With the wavelength interrogation method, we find that the proposed biosensor with two sensing channels exhibits a maximum refractive index sensitivity of 3400 $\mathrm{nm} / \mathrm{RIU}$ and a resolution of $2.94 \times 10^{-5}$ RIU for a large sensing range from 1.35 to 1.50 , which covers most known analytes of proteins, viruses or DNA/RNA. By utilizing 200 nm silver nanowires in the sensing channels, the sensitivity can be enhanced up to $4000 \mathrm{~nm} / \mathrm{RIU}$. Due to its special twochannel design for multi-sensing, it is possible to distinguish/study the binding possibility/capability of unknown analyte with two different target proteins simultaneously. Further, by introducing another critical channel, the confinement loss for either channel I or channel II can be greatly enhanced for high accurate result and more reliable sensing. Moreover, we numerically prove that the diameter of nano silver wires has great influences on the sensing peaks and sensitivity of the proposed biosensor.
\end{abstract}

Index Terms-D-shaped, Nano-sized metallic wire, Photonic Crystal Fibre, Refractive Index Sensor, Surface Plasmon Resonance, Sensitivity.

\section{INTRODUCTION}

$\mathbf{S}$ URFACE plasmon resonance (SPR) based fibre sensors have shown remarkable developments in numerous fields for label-free sensing application since they are highly robust and versatile optical tools. With the several outstanding advantages, in particular, high sensitivity, fast response, low-cost, capability of removable detection and real-time sensing, it now gains a considerable attention in a variety of research disciplines that range from genomics, proteomics, drug discovery,

Suoda Chu, K. Nakkeeran and Sumeet S. Aphale are with School of Engineering, Fraser Noble Building, University of Aberdeen, Aberdeen AB24 3UE, UK (email: r05sc15@abdn.ac.uk; K.Nakkeeran@abdn.ac.uk; S.Aphale@abdn.ac.uk).

Abdosllam M. Abobaker is with Department of Communications Engineering, College of Electronic Technology, Bani Walid, Libya (email: almahjub11@gmail.com).

S. Sivabalan is with School of Electrical Engineering, VIT University, Vellore-632 014, Tamil Nadu, India (email: ssivabalan@vit.ac.in).

P. Ramesh Babu and K. Senthilnathan are with Department of Physics, School of Advanced Sciences, VIT University, Vellore-632 014, Tamil Nadu, India (email: prameshbabu@vit.ac.in; senthee@gmail.com) food safety control, environment monitoring and development to medical diagnostics [1].

SPR is essentially an electromagnetic mode coupling phenomenon due to the collective resonant oscillation of free electrons in plasmonic metal, which is stimulated by $\mathrm{p}$ polarized incident light at the interface of metal and dielectric materials [2]. For a SPR based fibre sensor system, when the frequencies of incident light through the fibre and free electrons in the metal coated on the fibre are matched, the surface plasmons can be excited. This resonance condition is known as the SPR. Under this condition, a small portion of energy from the fundamental mode will be transferred to the surface plasmon mode, which in turn results in a dip in the fibre loss spectrum. Further, there would be a wavelength shift of the loss spectrum as and when the refractive index of the analyte is changed due to the surrounding environment [3]. It is also called propagating SPR as the enhanced surface plasmons will propagate along the thin metal film in the same direction of light propagation in the optical fibre.

Photonic crystal fibre (PCF), also called holey fibre or micro-structured fibre is a new class of special optical fibre. The cladding of such fibre consists of an arrangement of tiny and closely spaced air holes which go through the whole length of fibre [4]. It is possible to control the fibre optical characteristics by varying the diameter of the air holes and/or distance between the air holes and/or changing the arrangement of the air holes. Thus, the combination of PCF and SPR sensor technologies help in enhancing the sensitivity of the sensor by manipulating the structural parameters. In 2011, Zhou et al. proposed a PCF sensor based on silver nanocubes with a sensitivity of $3774.2 \mathrm{~nm} / \mathrm{RIU}$ [5]. In 2015, Otupuri et al. presented a novel PCF multi-channel biosensor with Gold and $\mathrm{Ta}_{2} \mathrm{TO}_{5}$ by measuring two fundamental modes [6]. Later, in 2017, Lu et al. demonstrated a multilayer-coated SPR sensor for dual refractive index range measurements with a capillary structure [7]. But PCF-SPR sensors in multi-sensing still suffer from low sensitivity or low contrast in the loss spectra of two analytes samples.

In this paper, we propose a six-fold dual core D-shaped PCF biosensor based on SPR phenomenon embedded with silver nanowires for multi-sensing and carry out the sensitivity analysis for a broad range of analytes of refractive index from 1.35 to 1.50 . Moreover, according to the loss spectra results in comparison with gold nanowires based biosensor, we find that silver nanowire is more suitable for multi-sensing due to 
low internal impact between the two resonance peaks from the coupling dynamics of the two channels for multi-sensing. Most importantly, the novel use of the critical channel with the additional silver nanowire increases the loss coefficient of the target analyte between two samples which can greatly reduce the measuring error for accurate and practical multi-sensing. The highest sensitivity can be obtained by applying $200 \mathrm{~nm}$ silver nanowires is $4000 \mathrm{~nm} / \mathrm{RIU}$. Therefore, the proposed biosensor has a steady and stable sensitivity characteristics and meet the requirements for multi-sensing with high efficiency that can be utilized as a biomedical tool for test-measurement of the molecular levels of biological samples or clinical identification of antigen/antibody for their binding reaction, etc.

\section{Geometric Structure And Theoretical MODELLING}

The cross sectional structure of the proposed SPR biosensor is shown in Fig. 1. The side polished depth of the dual core D-shaped PCF is set as $d=7.35 \mu \mathrm{m}$. Two half polished air holes are designed to fill in with nanowires and immobilized antibodies as sensing channels. Entire PCF-SPR sensor composed of four layers of air holes which are arranged in a fixed distance of lattice pitch, $\Lambda=2 \mu \mathrm{m}$. The diameter of air holes is set as $1.2 \mu \mathrm{m}$ and the diameter of silver nanowire is kept as $300 \mathrm{~nm}$. Those silver nanowires are embedded into the half polished air hole at the top surface, along the whole length of fibre sensor which is exactly between analyte and silica cladding for generating the SPR. Two different antibodies were immobilized around the silver nanowires within two sensing channels and the phosphate buffered saline (PBS) buffer solution with antigen flows through the biosensor's Dshaped surface. A three dimensional (3D) schematic of the proposed sensor is presented in Fig. 2 with silver nanowires, immobilized antibody and antigen in PBS buffer solution.

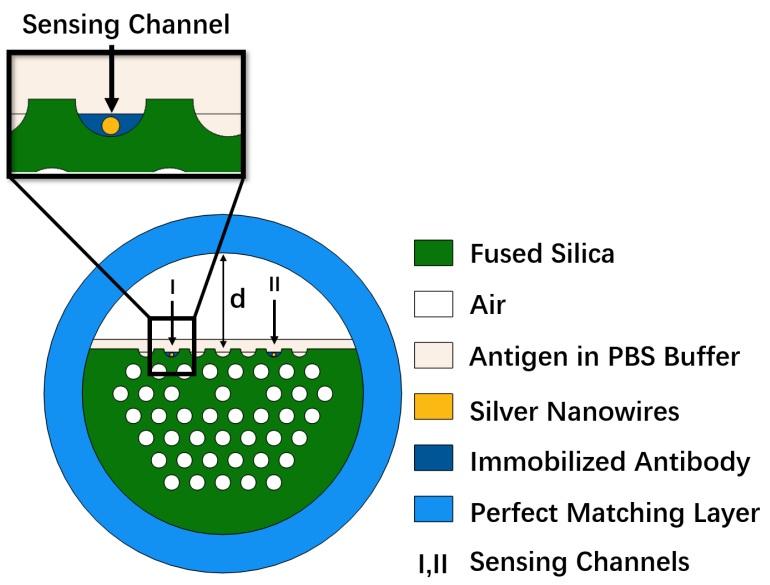

Fig. 1. Cross-section of the proposed six-fold dual core PCF-SPR biosensor.

In this structure, the cladding material is made of pure silica and the wavelength dependence of the refractive index of silica can be calculated from Sellmeier equation [8]:

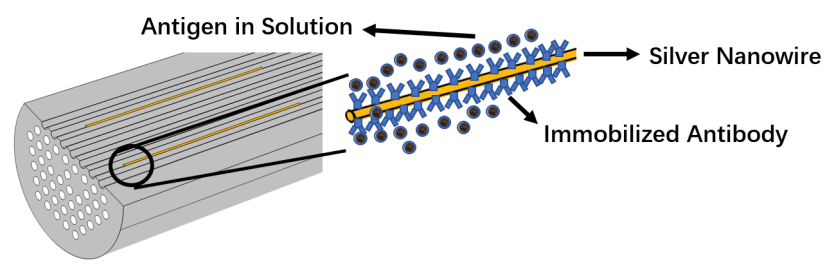

Fig. 2. 3D diagram of the proposed six-fold dual core PCF-SPR biosensor.

$$
n(\lambda)=\sqrt{1+\frac{B_{1}^{2}}{\lambda^{2}-C_{1}}+\frac{B_{2}{ }^{2}}{\lambda^{2}-C_{2}}+\frac{B_{3}^{2}}{\lambda^{2}-C_{3}}}
$$

where $\quad B_{1}=0.691663, \quad B_{2}=0.407943, \quad B_{3}=0.897479$, $C_{1}=0.004679, C_{2}=0.013512$, and $C_{3}=97.934003$. Here, $\lambda$ represents the incident light wavelength in vacuum. Following Drude model can be used to describe the dielectric constant of silver or gold [9]:

$$
\varepsilon(\lambda)=1-\frac{\lambda^{2} \lambda_{c}}{\lambda_{p}^{2}\left(\lambda_{c}+i \lambda\right)}
$$

where $\lambda_{p}$ is the plasma wavelength and $\lambda_{c}$ is the collision wavelength of the metal. For silver, $\lambda_{p}$ is $0.14541 \mu \mathrm{m}$ and $\lambda_{c}$ is $17.614 \mu \mathrm{m}$ [10]. In the case of gold, $\lambda_{p}$ is $0.16826 \mu \mathrm{m}$ and $\lambda_{c}$ is $8.9342 \mu \mathrm{m}$ [11]. As the binding process between the analyte and the protein/antibody surface results in an increase in the refractive index, the refractive indices in two sensing channels are considered as the channel I is fixed as 1.35 and channel II varies from 1.36 to 1.50 for comparison. The refractive index range is referenced from the research work of Voros [12]. The refractive index of the running PBS buffer solution with antigen/virus/DNA is considered as 1.34 [13]. For the numerical study, we use a finite element method (FEM) with a perfectly matched layer (PML) as the boundary condition.

The proposed dual core PCF biosensor can be fabricated by the state-of-art technique of stack-and-draw process [14] and side polishing method [15]. The stacked preform arrangement contains two solid fused silica rods for dual core and four layers of hollow silica capillaries for air holes in the fibre fabrication process as shown in Fig. 3. The well developed and controllable side polishing method is available to polish the desired depth for the D-shaped cross-section as required. Such D-shaped PCF with similar structure of half-open airhole channels was successfully fabricated by Kim et al., [16]. On the other hand, the metallic nanowire placement can be realized by filling nanowire colloids into the air-hole channels by capillary force and air pressure [17].

\section{AnAlysis of SENSING PERFormance}

For the proposed dual core PCF-SPR based biosensor with silver nanowires, due to the SPR phenomenon, it is well known that the energy of plasmon mode would arise as a consequence of the light energy transfer from the fundamental mode. Figures 4 illustrate both the distribution of electric field of the fundamental mode and the surface plasmon mode for a 


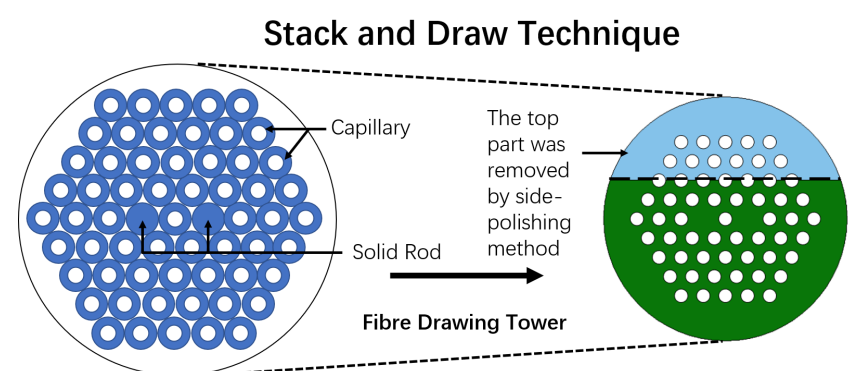

Fig. 3. Stacked preforms of the proposed dual-core PCF and side polishing method for the D-shaped surface with half-open air-hole channels.

range of wavelengths from $600 \mathrm{~nm}$ to $900 \mathrm{~nm}$. Here, the arrows indicate the direction of the electric field. It can be clearly seen from Figs. 4(a), 4(b) and 4(I) that a part of energy of coreguided fundamental mode from core area is transferred into plasmon mode around the nano-sized silver wire in channel I for $\mathrm{n}_{\mathrm{a}}=1.35$. Hence, the strongest surface plasmon resonance is excited as this energy transfer reaches to a maximum due to the phase matching between core-guided mode and plasmon mode occurs at this particular wavelength $(697 \mathrm{~nm})$. This phenomenon takes place as both the core-guided fundamental and the surface plasmon modes have the same real part of their propagation constants at this resonance wavelength. Hence, the intersection point of the dispersion relations for core-guided fundamental mode and plasmon mode can be used to locate the resonance wavelength. Results in Figs. 4(b), 4(c) and 4(II), depict another resonance for $\mathrm{n}_{\mathrm{a}}=1.38$ when the wavelength approaches to $780 \mathrm{~nm}$ in channel II. As the monoclonal antibody binds only to one ligand site of a particular antigen due to the specific binding principle [18], the two channels will have no influence with each other as long as their refractive indices of binding layer are different. This helps the biosensor to achieve highly accurate and stable sensing results. Finally, results presented in Figs. 4(c) and 4(d), show that the wavelength of the incident light is not creating any considerable resonance, as the energy can be barely transferred to the surface plasmon mode. Owing to a shift in the confinement loss peak of the resonance wavelength for analytes of different refractive indices, the minute change of surrounding refractive index can be detected. Therefore, we can make use of those confinement loss peaks for multi-sensing purpose. Moreover, because of the association and dissociation in the process of antigen-antibody binding reaction, this biosensor can also be used to monitor the reaction duration by detecting their SPR refractive index changes based on the confinement loss data over time scale. According to [19], the confinement loss $\alpha_{\text {loss }}$ is defined by:

$$
\alpha_{\text {loss }}=\frac{40 \pi}{\lambda \ln 10} \Im\left(n_{\text {eff }}\right),
$$

where $\lambda$ is the wavelength of incident light in vacuum and $\Im\left(n_{\text {eff }}\right)$ represents the imaginary part of the effective refractive index of the core-guided fundamental mode.

The dispersion relation of the core-guided fundamental mode and surface plasmonic mode along with the confinement loss is presented in Fig. 5 when the refractive indices of

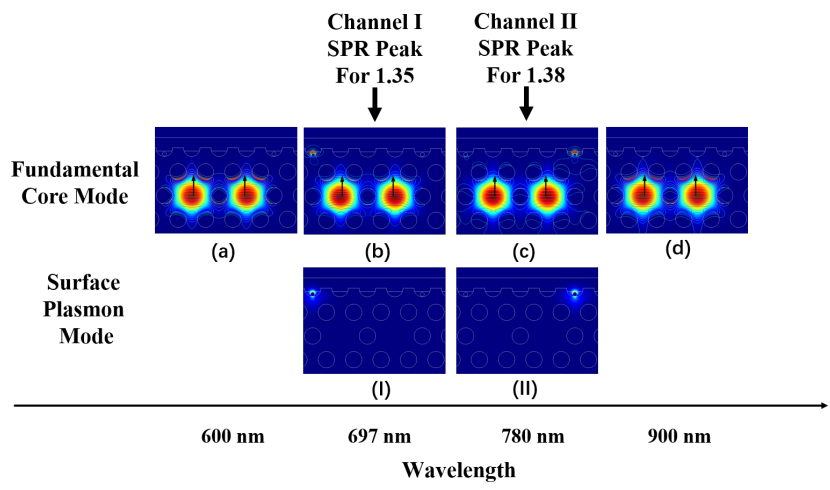

Fig. 4. Light energy flow distributions in the cross-section of dual core Dshaped PCF-SPR sensor for different wavelengths for the analyte refractive index of $\mathrm{n}_{\mathrm{a}}=1.35$ and $\mathrm{n}_{\mathrm{a}}=1.38$. (a) is the fundamental mode at $600 \mathrm{~nm}$. (b) and (I) are the fundamental mode and surface plasmonic mode at first resonance wavelength of $697 \mathrm{~nm}$ for $n_{a}=1.35$ of channel I. (c) and (II) are the fundamental mode and plasmonic mode at the second resonance wavelength of $780 \mathrm{~nm}$ for $\mathrm{n}_{\mathrm{a}}=1.38$ of channel II. (d) is the fundamental mode at 900 $\mathrm{nm}$. The arrows indicate the direction of the electric field.

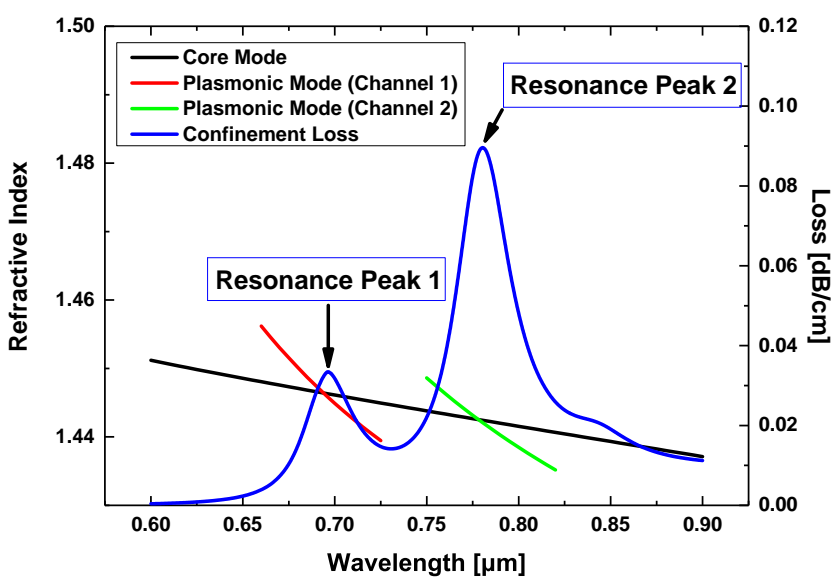

Fig. 5. The dispersion relation of the fundamental mode (black line), two surface plasmon modes for $\mathrm{n}_{\mathrm{a}}=1.35$ (red line, channel $\mathrm{I}$ ) and $\mathrm{n}_{\mathrm{a}}=1.38$ (green line, channel II) at $697 \mathrm{~nm}$ and $780 \mathrm{~nm}$, respectively and confinement loss (blue line) for wavelength range from $600 \mathrm{~nm}$ to $900 \mathrm{~nm}$.

antigen-antibody bindings are 1.35 and 1.38 . It is obvious that the confinement loss approaches maximum at the intersection point of core mode and plasmonic mode due to the maximum energy leakage of fundamental mode at the resonance wavelength. As discussed before, when the real part of the effective refractive index of the fundamental mode coincide with the two SPR modes (red and green lines in Fig. 5), the phase matching conditions for two coupling dynamics between the two sensing channels take place at $697 \mathrm{~nm}$ and $780 \mathrm{~nm}$, respectively.

For a refractive index based biosensor, the SPR wavelength is extremely sensitive to the surrounding refractive index changes. The variations of loss spectra against wavelength are presented in Fig. 6 for various analytes of refractive index varying from 1.35 to 1.45 . The detailed sensitivity data is presented in Table. I for a full set of analyte of refractive index from 1.35 to 1.50 . From the simulation results, it is obvious that as the refractive index of the analyte increases, the loss 


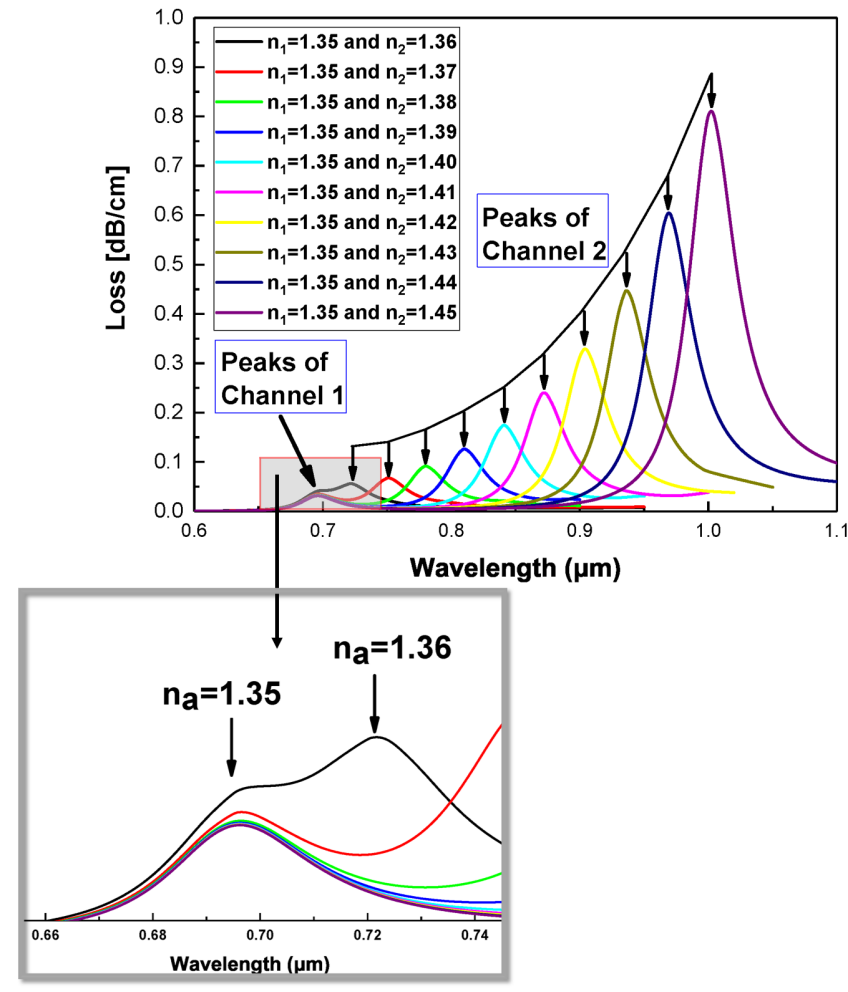

Fig. 6. Confinement loss spectra for $\mathrm{n}_{\mathrm{a}}$ of channel I fixed at 1.35 while channel II is set as a range of $n_{a}$ from 1.36 to 1.45 for comparison. The enlarged region shows the details of the peaks of $n_{a}=1.35$ in channel I and the peak of $n_{a}=1.36$ in channel II.

peak is getting shifted towards the longer wavelength with increase in their respective loss amplitudes. The enlarged graph shows that for the detection of $n_{a}=1.35$ and 1.36 , due to the sub-peak influence [20], the two loss curves become broader and blunt, making it difficult to detect. Hence, according to the loss spectra, the detectable analytes refractive index threshold for multi-sensing is limited to a resolution of 0.01. Also, from the simulation results, the maximum sensitivity is computed to be $3400 \mathrm{~nm} / \mathrm{RIU}$ using the wavelength interrogation method [21]. For a $0.1 \mathrm{~nm}$ peak-wavelength resolution instrument, the refractive index resolution of corresponding sensor is $2.94 \times 10^{-5}$ RIU. How much sharp/blunt (narrow/broad) the confinement loss characteristics is indicated by the full-width at half-maximum (FWHM) bandwidth of the the curve and for $\mathrm{n}_{\mathrm{a}}=1.36$, the loss curve is too blunt and broad to be calculated (mentioned as N/A in Table. I). The average figure of merit (FOM) value for the proposed biosensor is calculated to be 74.5 $\mathrm{RIU}^{-1}$.

By swapping the silver nanowires immobilized with antibodys, which corresponds to the change of refractive indices of the binding layers to 1.38 for channel I and 1.35 for channel II, we find that the loss spectra remain the same as shown in Fig. 5. This proves that the resonant characteristics of this biosensor with such nanowire configuration are not related to the channel position but it is determined by the refractive indices of the binding layers. On the other hand, this inference brings the limitation on the application of the proposed biosensor to distinguish the target binding analytes
TABLE I

SENSITIVITY DATA OF THE D-SHAPED DUAL CORE PCF-SPR BIOSENSOR

(BINDING LAYER RANGES FROM 1.35 TO 1.50 [SILVER NANOWIRE])

\begin{tabular}{lcccc}
$\begin{array}{l}\text { Analyte } \\
\text { Refractive } \\
\text { Index }\end{array}$ & $\begin{array}{c}\text { Resonant } \\
\text { Wavelength } \\
(\mathrm{nm})\end{array}$ & $\begin{array}{c}\text { Peak } \\
\text { Loss } \\
(\mathrm{dB} / \mathrm{cm})\end{array}$ & $\begin{array}{c}\text { FWHM } \\
\text { Bandwidth } \\
(\mathrm{nm})\end{array}$ & $\begin{array}{c}\text { Sensitivity } \\
(\mathrm{nm} / \mathrm{RIU})\end{array}$ \\
\hline & & & & \\
1.35 & 697 & 0.030 & 37.2 & - \\
1.36 & 722 & 0.056 & N/A & 2500 \\
1.37 & 751 & 0.068 & 38.5 & 2900 \\
1.38 & 780 & 0.092 & 37.5 & 3000 \\
1.39 & 810 & 0.126 & 38.3 & 3100 \\
1.40 & 841 & 0.174 & 39.4 & 3100 \\
1.41 & 872 & 0.240 & 40.4 & 3200 \\
1.42 & 904 & 0.329 & 41.7 & 3200 \\
1.43 & 936 & 0.447 & 42.9 & 3200 \\
1.44 & 969 & 0.605 & 44.2 & 3300 \\
1.45 & 1002 & 0.811 & 45.5 & 3300 \\
1.46 & 1036 & 1.080 & 46.5 & 3400 \\
1.47 & 1069 & 1.429 & 47.6 & 3300 \\
1.48 & 1103 & 1.877 & 48.6 & 3400 \\
1.49 & 1137 & 2.448 & 49.7 & 3400 \\
1.50 & 1170 & 3.171 & 49.1 & 3400
\end{tabular}

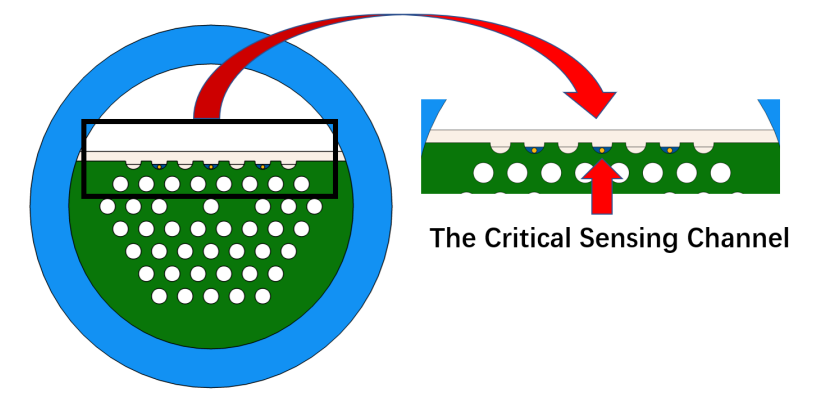

Fig. 7. Cross-section of the D-shaped dual core PCF-SPR biosensor with critical channel of silver nanowires.

between the two channels simultaneously. Moreover, as listed in Table. I, the confinement losses are less than $0.1 \mathrm{~dB} / \mathrm{cm}$ for a range of analyte refractive indices from 1.35 to 1.38 . Those confinement loss values will cause lower power loss that cannot be detected appropriately and accurately using an optical spectrum analyzer (OSA). In order to overcome those limitations, a critical channel with an addition silver nanowire is introduced in the central half-polished air hole of D-shaped surface, as illustrated in Fig. 7. Three different scenarios are considered for comparison of this modified biosensor: (1) critical channel with $n_{a}=1.35$, (2) no critical channel and (3) critical channel with $n_{a}=1.38$. The results reported in Fig. 8 show that with a single nanowire (either $n_{a}=1.35$ or 1.38) present in the critical channel, the confinement loss gets significant enhancement than the case without any critical channel. For RI values of $n_{a}=1.35$ and 1.38 , the confinement losses can be increased from $0.03 \mathrm{~dB} / \mathrm{cm}$ to $0.157 \mathrm{~dB} / \mathrm{cm}$ and $0.092 \mathrm{~dB} / \mathrm{cm}$ to $0.445 \mathrm{~dB} / \mathrm{cm}$, respectively. Typically, PCFSPR biosensors require only a short length (L) of optical fibre (usually $\mathrm{L}=10 \mathrm{~mm}$ to $25 \mathrm{~mm}$ ) [22]. Hence, for a 10 $\mathrm{mm}$ long PCF-SPR biosensor, the minimum transmission loss could be $0.157 \mathrm{~dB}$ for a RI of 1.35 and $0.445 \mathrm{~dB}$ for a RI of 


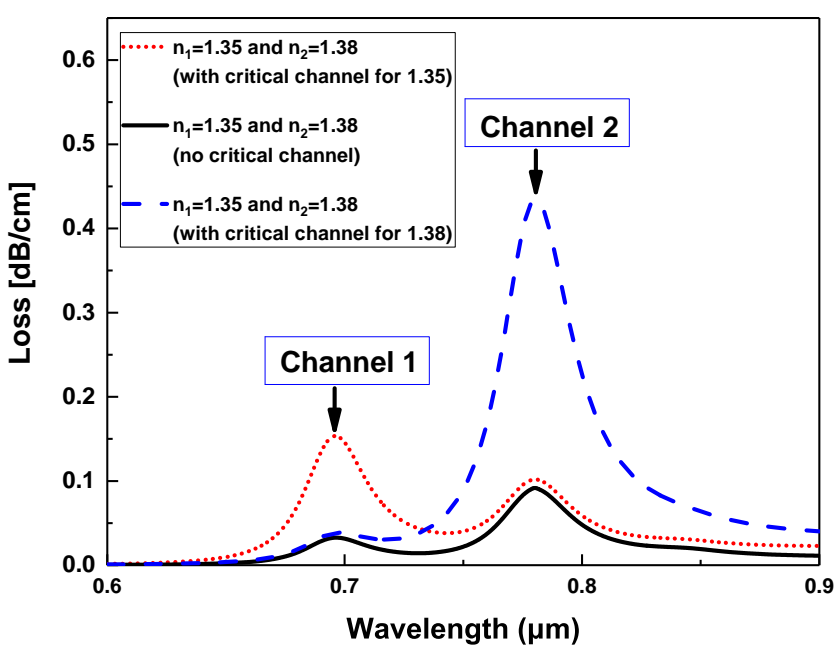

Fig. 8. The loss spectra as a function of wavelength when the refractive index of binding layers are set as 1.35 and 1.38 for multi-sensing enhanced by critical channel with an additional silver nanowire.

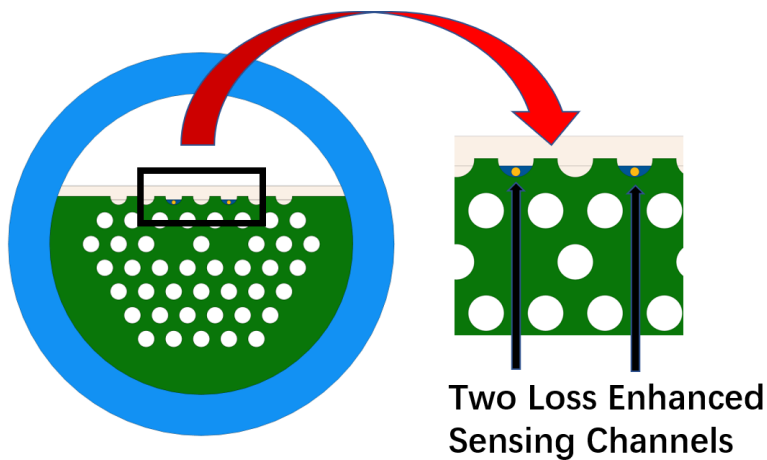

Fig. 9. Cross-section of the D-shaped dual core PCF-SPR biosensor with two loss enhanced channels of silver nanowires.

1.38 , which is $3.55 \%$ and $9.74 \%$ variation in the transmitted intensities, respectively. According to [22], the power loss of the enhanced biosensor can meet the requirement of at least $1 \%$ variation in the transmitted intensity with distinguishable loss peak for proper sensing. Also, due to the low confinement loss characteristics of the proposed PCF-SPR biosensor it might be required to use a lengthier PCF than the typical value to achieve the minimum required power loss.

Another way to achieve more distinguishable loss peak (confinement loss enhancement) for better sensing is to use a PCF-SPR biosensor design structure as shown in Fig. 9. This D-shaped dual core PCF-SPR biosensor with two channels of silver nanowires configuration exhibits a significant confinement loss enhancement as shown in Fig. 10. For a RI value of $n_{a}=1.38$, the confinement loss can reach up to $4.728 \mathrm{~dB} / \mathrm{cm}$, which is 50 times more than the first proposed D-shaped configuration (Fig. 1), and 10 times more than the configuration with critical channel (Fig. 7). With the enhanced confinement loss characteristic (Fig. 10) of the two-channels PCF-SPR biosensor with nanowires (Fig. 9), it is possible to keep the length of the proposed biosensors within the typical value of the sensing region. However, further improvement of the two-channel nanowires configuration by adding another

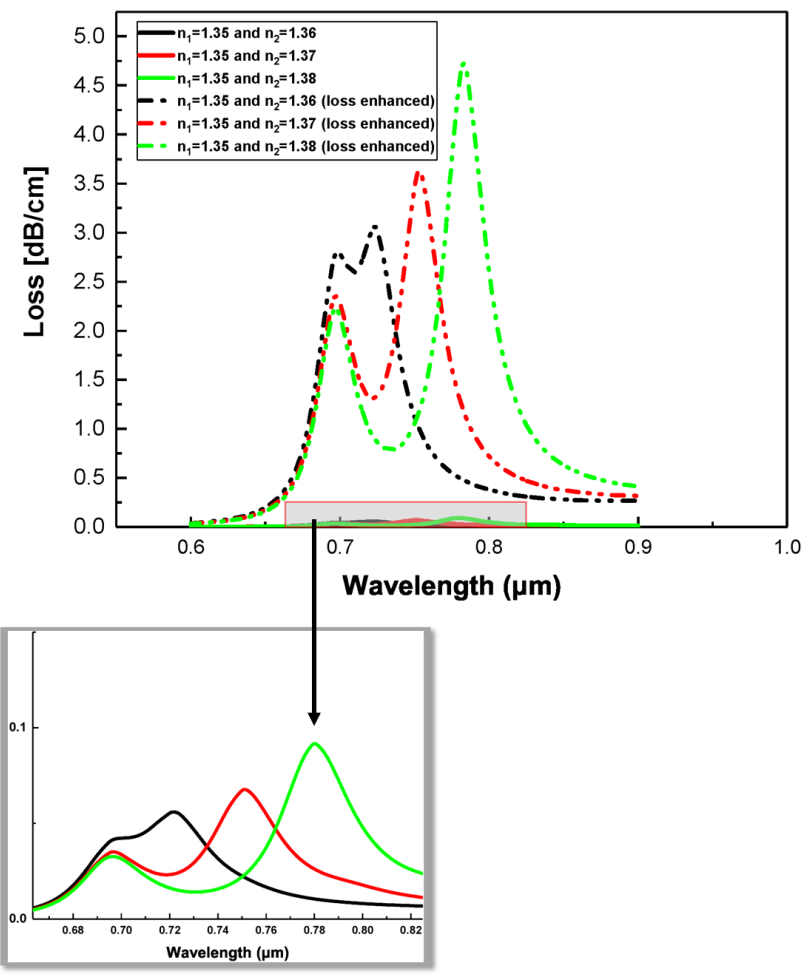

Fig. 10. The comparison of loss spectra as a function of wavelength when the refractive index of binding layers are set as 1.35 and 1.38 for multi-sensing with (solid lines) and without (dashed lines) loss enhanced channels.

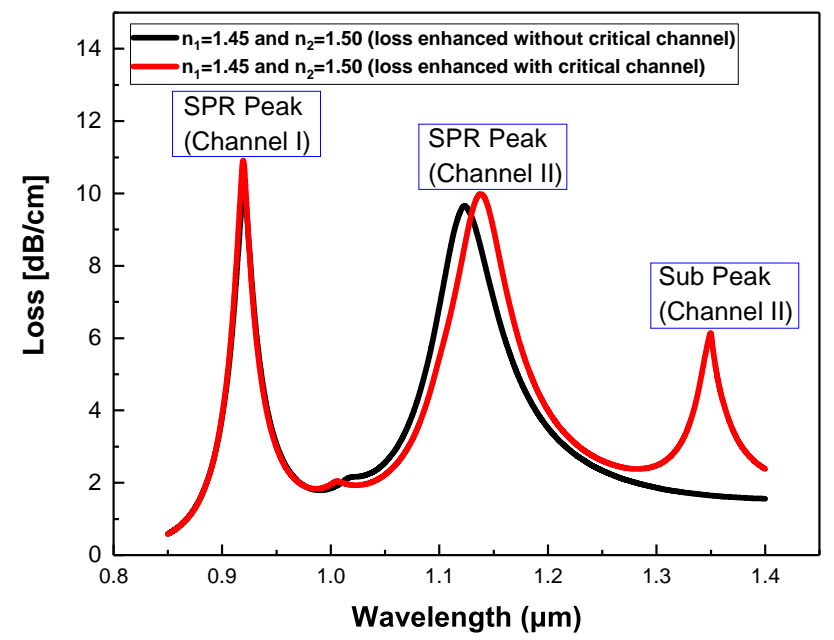

Fig. 11. The loss spectra as a function of wavelength when the refractive indices of binding layers are set as 1.45/1.50 in the confinement loss enhanced configuration with and without the critical channel (nanowire diameter $=200$ $\mathrm{nm})$.

critical channel with nanowire for multi-sensing to distinguish the binding reactions in two different channels will not be possible. It is because two adjacent nanowires in the configuration would cause two surface plasmons for higher refractive index analyte, especially when the diameter size of the nanowires are very small. The unwanted sub-peak of SPR illustrated in Fig. 11 might affect the accuracy and stability of the PCF-SPR biosensor [20].

Gold (noble metal with higher chemical stability) is the most commonly used material in practice for SPR sensor designs 


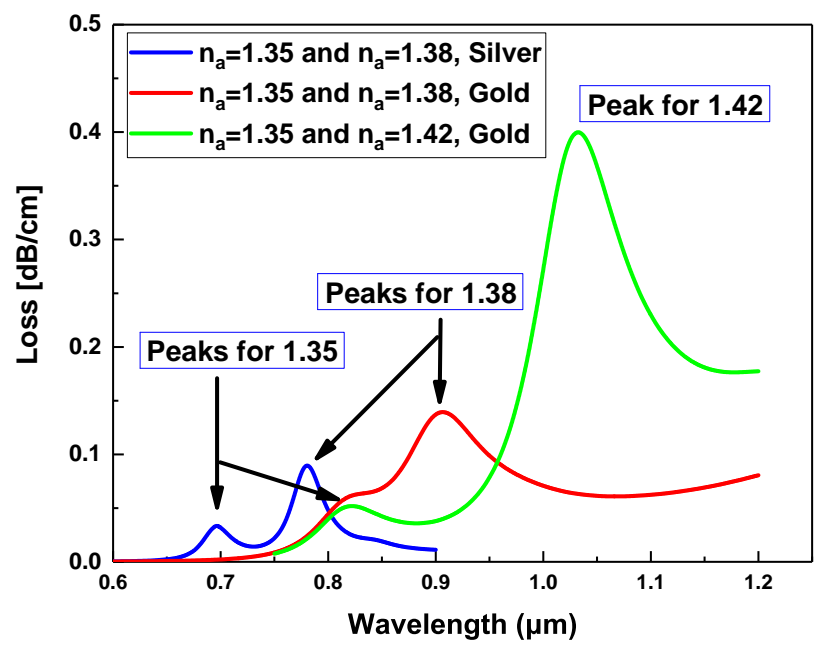

Fig. 12. The loss spectra as a function of wavelength when the refractive indices of binding layers are set as 1.35/1.38 and 1.35/1.42 with gold nanowires compared with silver nanowires for $\mathrm{n}_{\mathrm{a}}=1.35 / 1.38$.

compared to silver because of its chemical susceptibility to oxidation. For comparison, we investigate the sensing performance of gold nanowires embedded in the sensing channels instead of silver nanowires in the two-channel configuration of the biosensor and its dielectric constant can be calculated using Drude model as shown in Eq. 2. In contrast to the sensing performance of silver nanowires, gold nanowires show broader (comparatively large values of FHMW bandwidth) loss characteristics due to higher ohmic losses. Simulation results in Fig. 12 indicate that the biosensor with gold nanowires exhibits both higher confinement and contrast for the loss characteristics between the analytes, but failed to show sharp curves/peaks for 1.35 in both multi-sensing tests (other channel $\mathrm{n}_{\mathrm{a}}=1.38$ or 1.42 ) as the bluntness in the loss spectra are caused by the influence of the sub-peak [20]. Hence, we conclude that the proposed biosensor with silver nanowires has better sensing performance over gold nanowires for multi-sensing.

\section{INFLUENCE AND OPTIMIZATION OF VARIATIONS IN STRUCTURAL PARAMETERS}

It is well know that the resonance wavelength of SPR is highly dependent on the dimension of the metal nanowires [23]. Hence, it is possible to modify the SPR resonance wavelength simply by fabricating the desired diameter size of nanowires. Here, we investigate how different diameters of the silver nanowires influence the sensing performances of the designed biosensor. As illustrated in Fig. 13, by varying the diameter of silver nanowires, $D_{w}$, the resonance wavelengths of the biosensor with two analytes have obvious shifts compared to the one with the $300 \mathrm{~nm}$ silver nanowires in twochannel configuration as reference. For larger diameter silver nanowires $(350 \mathrm{~nm})$, a redshift is noticed in the confinement loss spectra. On the contrary, for silver nanowires with smaller diameters $(200 \mathrm{~nm}$ and $250 \mathrm{~nm}$ ), we find that the confinement loss spectra have blueshifts. Table. II shows all the resonant wavelengths for different diameters of silver nanowires for a range of refractive index of $1.35-1.50$. The calculated sensitivity reveals enhancement up to $4000 \mathrm{~nm} / \mathrm{RIU}$ from 200 $\mathrm{nm}$ silver nanowires, whereas the maximum sensitivity of 350 $\mathrm{nm}$ silver nanowires configuration decreases to $3000 \mathrm{~nm} / \mathrm{RIU}$. Moreover, the R-squared values illustrated in Fig. 14 indicate that this biosensor has a good linear response for its sensing performances.

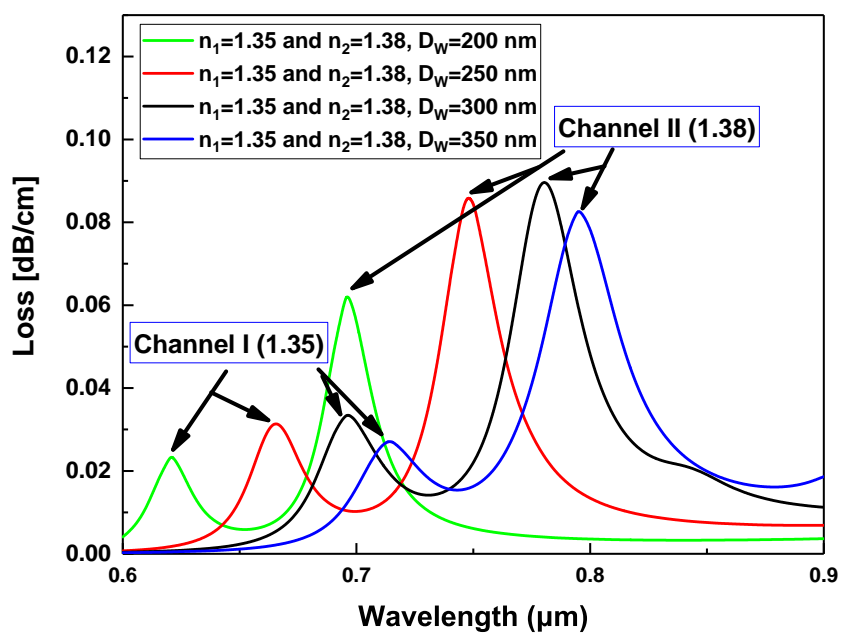

Fig. 13. The loss spectra as a function of wavelength when the refractive indices of binding layers are set as 1.35 and 1.38. Diameter of the silver nanowire: $200 \mathrm{~nm}, 250 \mathrm{~nm}, 300 \mathrm{~nm}$ and $350 \mathrm{~nm}$. ( $D_{\mathrm{w}}=300 \mathrm{~nm}$ as reference).

Apart from the diameter of the nanowire, the distance between the air holes $(\Lambda$, also called pitch) and air hole diameters $\left(D_{a}\right)$ are another important structural parameters for the PCF-SPR biosensor that have great influence on the optical characteristics and sensing performances. Figures 15 and 16 show the confinement loss spectra for different pitches and air hole diameters for analytes in the binding layers with refractive index as 1.35 and 1.38 , respectively. The solid line indicates the referenced structural parameters $(\Lambda=2 \mu \mathrm{m})$ and the dotted and dashed lines represent the values lower $(\Lambda=1.9 \mu \mathrm{m})$ and higher $(\Lambda=2.1 \mu \mathrm{m})$ than the referenced one. Note an increase in the confinement loss spectra with a blueshift as shown by the dashed line in Fig. 15 when the pitch is increased by $5 \%$ and vice-versa as shown by the dotted line in Fig. 15 when the pitch is decreased by $5 \%$.

Next, we study the performance of the biosensor by varying the diameter of the air holes. As shown in Fig. 16, the confinement loss spectra undergo blueshift and increase in their values when the diameter of the air hole is decreased by $5 \%$ from the referenced value $\left(D_{a}=1.2 \mu \mathrm{m}\right)$. However, there is no significant wavelength shift when the air holes diameter increased by $5 \%$, but there is decrease in the confinement loss values. The difference between resonant peaks (main and sub peaks of the same air hole size) wavelength increases as the air hole diameter increases.

By investigating the structural parameters, we find that the sensing performances of the proposed biosensor will be influenced by the variations of the nanowires diameter, air holes diameter and pitch. Significant influence on the biosensor sensitivity is due to the decreasing size of the silver nanowire diameter. Moreover, for different values of air hole diameter or pitch values, affect both the magnitudes of the loss spectra 
TABLE II

The Resonant WaVELENGTH OF THE D-ShaPed DUAL CORE PCF-SPR BIOSENSOR

(BINDING LAYER RANGES FROM 1.35 TO 1.50 [SILVER NANOWIRE])

\begin{tabular}{lcccc}
$\begin{array}{l}\text { Analyte } \\
\text { Refractive } \\
\text { Index }\end{array}$ & $\begin{array}{c}200 \mathrm{~nm} \\
\text { Silver } \\
\text { Nanowires } \\
(\mathrm{nm})\end{array}$ & $\begin{array}{c}250 \mathrm{~nm} \\
\text { Silver } \\
\text { Nanowires } \\
(\mathrm{nm})\end{array}$ & $\begin{array}{c}300 \mathrm{~nm} \\
\text { Silver } \\
\text { Nanowires } \\
(\mathrm{nm})\end{array}$ & $\begin{array}{c}350 \mathrm{~nm} \\
\text { Silver } \\
\text { Nanowires } \\
(\mathrm{nm})\end{array}$ \\
\hline & & & & \\
1.35 & 621 & 665 & 697 & 715 \\
1.36 & 644 & 691 & 722 & 739 \\
1.37 & 669 & 719 & 751 & 768 \\
1.38 & 696 & 748 & 780 & 795 \\
1.39 & 724 & 778 & 810 & 824 \\
1.40 & 754 & 809 & 841 & 853 \\
1.41 & 785 & 841 & 872 & 883 \\
1.42 & 818 & 874 & 904 & 911 \\
1.43 & 852 & 908 & 936 & 941 \\
1.44 & 887 & 943 & 969 & 970 \\
1.45 & 923 & 978 & 1002 & 999 \\
1.46 & 961 & 1015 & 1036 & 1029 \\
1.47 & 1000 & 1051 & 1069 & 1058 \\
1.48 & 1039 & 1088 & 1103 & 1087 \\
1.49 & 1079 & 1126 & 1137 & 1115 \\
1.50 & 1119 & 1163 & 1171 & 1144
\end{tabular}

and the resonant wavelengths of the biosensor. This is because of the coupling dynamics that happens between the sensing channels. So the sensing performance will largely depend on the sensing channels' structural design parameters, which are nanowires diameter, air hole diameter and pitch (distance between the air holes). Therefore, a careful calibration involving all these PCF design parametes is very important for practical use of this biosensor.

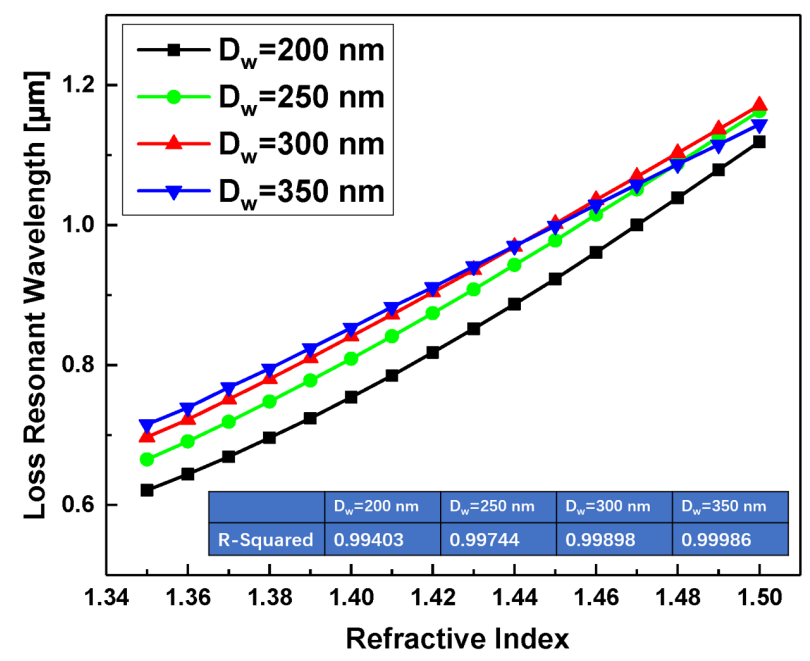

Fig. 14. Loss resonant wavelengths versus analyte refractive index with Rsquared values.

\section{CONCLUSION}

In this paper, we have proposed a D-shaped dual core sixfold PCF-SPR sensor embedded with silver nanowires for multi-sensing through two-channel configuration. A maximum wavelength sensitivity of $3400 \mathrm{~nm} / \mathrm{RIU}$ and a resolution of $2.94 \times 10^{-5}$ RIU have been achieved for a sensing refractive

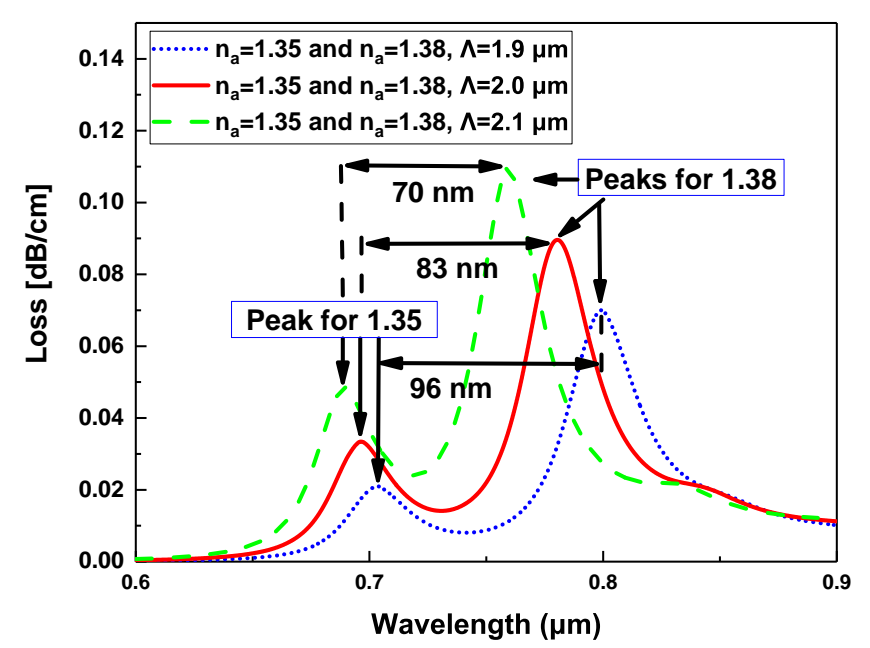

Fig. 15. The loss spectra as a function of wavelength when the refractive indices of binding layers are set as 1.35 and 1.38 with three different pitch values, $1.9 \mu \mathrm{m}(-5 \%), 2.0 \mu \mathrm{m}$ (reference), $2.1 \mu \mathrm{m}(+5 \%)$.

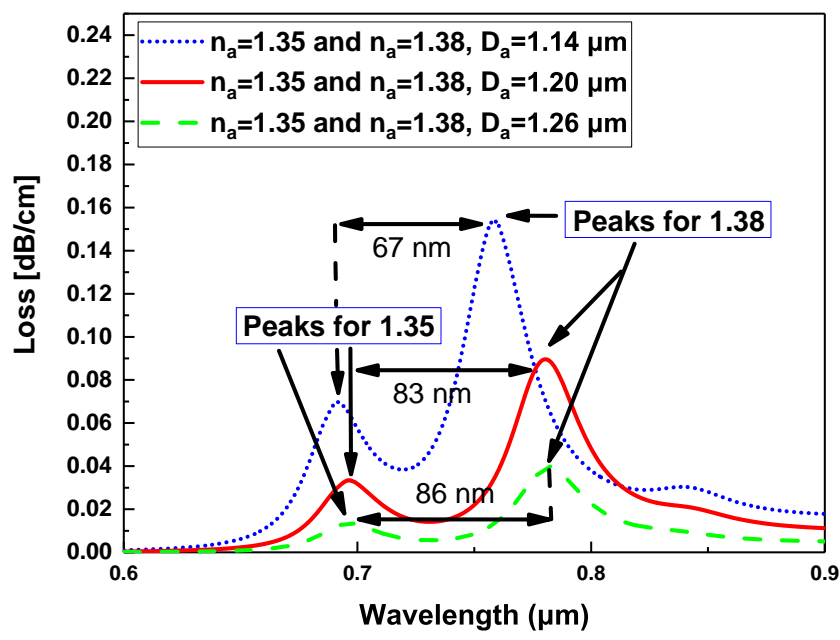

Fig. 16. The loss spectra as a function of wavelength when the refractive indices of binding layers are set as 1.35 and 1.38 with three different air holes diameter values, $1.14 \mu \mathrm{m}(-5 \%), 1.2 \mu \mathrm{m}$ (reference) and $1.26 \mu \mathrm{m}(+5 \%)$.

index range of binding layer from 1.35 to 1.50 with $300 \mathrm{~nm}$ diameter silver nanowires. Further, by decreasing the diameter of the nanowires to $200 \mathrm{~nm}$, it is possible to achieve a higher sensitivity value of $4000 \mathrm{~nm} / \mathrm{RIU}$. Although in comparison, this amount of maximum sensitivity is not as high as the earlier reported biosensor designs [4], [20], the biosensor presented in this work can achieve larger refractive index sensing range with appreciable and stable sensitivity. According to the simulation results, proposed biosensor design with silver nanowires are more suitable for multi-sensing than gold nanowires due to low sub-peak influence. Most importantly, it has been found that, by adding a critical channel with silver nanowire, this novel PCF-SPR biosensor design can distinguish the target analytes of binding reactions between two samples simultaneously. Resonant peak wavelengths (position and separation between the main and sub peaks) and the magnitude of the confinement loss of the proposed biosensor could be significantly influenced by the variations of the PCF structural 
design parameters like the diameter of the nanowires, air hole diameter and pitch (distance between the air holes). In a conclusion, we believe that the proposed biosensor would be highly efficient in multi-sensing of the refractive index changes of different analytes in the binding layers. Due to its simple design structure, it can be fabricated easily by side-polishing process. Based on the above comprehensive discussion and analysis, we are of the opinion that the proposed novel sensor would be a promising candidate for biochemical detections.

\section{REFERENCES}

[1] E. P. Rodrigues, A. M. N. Lima, L. C. Oliveira, T. A. T. de Sousa, and H. Neff, "Surface plasmon resonance features of corrugated gold films: wavelength interrogation mode for exhaled gas detection," Journal of Physics: Conference Series, vol. 1044, p. 012062, jun 2018 [Online]. Available: https://doi.org/10.1088\%2F1742-6596\%2F1044\% 2F1\%2F012062

[2] G. An, S. Li, X. Yan, X. Zhang, Z. Yuan, H. Wang, Y. Zhang, X. Hao, Y. Shao, and Z. Han, "Extra-broad photonic crystal fiber refractive index sensor based on surface plasmon resonance," Plasmonics, vol. 12, no. 2, pp. 465-471, 2017.

[3] M. S. A. Gandhi, S. Chu, K. Senthilnathan, P. R. Babu, K. Nakkeeran, and Q. Li, "Recent advances in plasmonic sensor-based fiber optic probes for biological applications," Applied Sciences, vol. 9, no. 5, 2019. [Online]. Available: https://www.mdpi.com/2076-3417/9/5/949

[4] S. Chu, K. Nakkeeran, A. M. Abobaker, S. S. Aphale, P. R. Babu, and K. Senthilnathan, "Design and analysis of surface-plasmon-resonancebased photonic quasi-crystal fiber biosensor for high-refractive-index liquid analytes," IEEE Journal of Selected Topics in Quantum Electronics, vol. 25, no. 2, pp. 1-9, March 2019.

[5] C. Zhou, "Localized surface plasmonic resonance study of silver nanocubes for photonic crystal fiber sensor," Optics and Lasers in Engineering, vol. 50, no. 11, pp. 1592 - 1595, 2012. [Online]. Available: http://www.sciencedirect.com/science/article/pii/S0143816612001613

[6] R. Otupiri, E. K. Akowuah, and S. Haxha, "Multi-channel spr biosensor based on pcf for multi-analyte sensing applications," Opt. Express, vol. 23, no. 12, pp. 15716-15727, Jun 2015. [Online]. Available: http://www.opticsexpress.org/abstract.cfm?URI=oe-23-12-15716

[7] M. Lu, W. Peng, Q. Liu, Y. Liu, L. Li, Y. Liang, and J.-F. Masson, "Dual channel multilayer-coated surface plasmon resonance sensor for dual refractive index range measurements," Opt. Express, vol. 25, no. 8, pp. 8563-8570, Apr 2017. [Online]. Available: http://www.opticsexpress.org/abstract.cfm?URI=oe-25-8-8563

[8] E. K. Akowuah, T. Gorman, H. Ademgil, S. Haxha, G. K. Robinson, and J. V. Oliver, "Numerical analysis of a photonic crystal fiber for biosensing applications," IEEE Journal of Quantum Electronics, vol. 48, no. 11, pp. 1403-1410, 2012.

[9] N. Zainuddin, M. Ariannejad, P. Arasu, S. Harun, and R. Zakaria, "Investigation of cladding thicknesses on silver spr based side-polished optical fiber refractive-index sensor," Results in Physics, vol. 13 , p. 102255, 2019. [Online]. Available: http://www.sciencedirect.com/ science/article/pii/S2211379719304954

[10] R. Tabassum and B. D. Gupta, "Surface plasmon resonance-based fiber-optic hydrogen gas sensor utilizing palladium supported zinc oxide multilayers and their nanocomposite," Appl. Opt., vol. 54, no. 5, pp. 1032-1040, Feb 2015. [Online]. Available: http://ao.osa.org/abstract. cfm?URI=ao-54-5-1032

[11] A. K. Mishra, S. K. Mishra, and R. K. Verma, "Graphene and beyond graphene mos2: A new window in surface-plasmon-resonancebased fiber optic sensing," The Journal of Physical Chemistry $C$, vol. 120, no. 5, pp. 2893-2900, 2016. [Online]. Available: https://doi.org/10.1021/acs.jpcc.5b08955

[12] J. Vrs, "The density and refractive index of adsorbing protein layers," Biophysical Journal, vol. 87, no. 1, pp. 553 - 561 , 2004. [Online]. Available: http://www.sciencedirect.com/science/article/ pii/S0006349504735407

[13] L. Diéguez, N. Darwish, M. Mir, E. Martínez, M. Moreno, and J. Samitier, "Effect of the refractive index of buffer solutions in evanescent optical biosensors," Sensor Letters, vol. 7, no. 5, 2009.

[14] E. Chillcce, C. Cordeiro, L. Barbosa, and C. B. Cruz, "Tellurite photonic crystal fiber made by a stack-and-draw technique," Journal of Non-Crystalline Solids, vol. 352, no. 32, pp. 3423 - 3428,
2006, glasses and Related Materials 7. [Online]. Available: http: //www.sciencedirect.com/science/article/pii/S0022309306007654

[15] S.-M. Tseng and C.-L. Chen, "Side-polished fibers," Appl. Opt., vol. 31, no. 18, pp. 3438-3447, Jun 1992. [Online]. Available: http://ao.osa.org/abstract.cfm?URI=ao-31-18-3438

[16] H. Kim, J. Kim, U.-C. Paek, B. H. Lee, and K. T. Kim, "Tunable photonic crystal fiber coupler based on a side-polishing technique," Opt. Lett., vol. 29, no. 11, pp. 1194-1196, Jun 2004. [Online]. Available: http://ol.osa.org/abstract.cfm?URI=ol-29-11-1194

[17] X. C. Yang, Y. Lu, B. L. Liu, and J. Q. Yao, "Temperature sensor based on photonic crystal fiber filled with liquid and silver nanowires," IEEE Photonics Journal, vol. 8, no. 3, pp. 1-9, June 2016.

[18] E. Suraniti, E. Sollier, R. Calemczuk, T. Livache, P. N. Marche, M.-B. Villiers, and Y. Roupioz, "Real-time detection of lymphocytes binding on an antibody chip using spr imaging," Lab Chip, vol. 7, pp. 12061208, 2007. [Online]. Available: http://dx.doi.org/10.1039/B708292D

[19] P.-p. Zhang, J.-q. Yao, H.-x. Cui, and Y. Lu, "A surface plasmon resonance sensor based on a multi-core photonic crystal fiber," Optoelectronics Letters, vol. 9, no. 5, pp. 342-345, 2013.

[20] S. Chu, K. Nakkeeran, A. M. Abobaker, S. S. Aphale, S. Sivabalan, P. R. Babu, and K. Senthilnathan, "Influence of the sub-peak of secondary surface plasmon resonance onto the sensing performance of a d-shaped photonic crystal fibre sensor," IEEE Sensors Journal, pp. 1-1, 2019.

[21] J. Homola, S. S. Yee, and G. Gauglitz, "Surface plasmon resonance sensors: review," Sensors and Actuators B: Chemical, vol. 54, no. 1, pp. 3 - 15, 1999. [Online]. Available: http://www.sciencedirect.com/ science/article/pii/S0925400598003219

[22] A. Hassani and M. Skorobogatiy, "Design of the microstructured optical fiber-based surface plasmon resonance sensors with enhanced microfluidics," Opt. Express, vol. 14, no. 24, pp. 11616-11 621, Nov 2006. [Online]. Available: http://www.opticsexpress.org/abstract.cfm? URI=oe-14-24-11616

[23] H. Lee, H.-J. Kim, J.-H. Park, D. H. Jeong, and S.-K. Lee, "Effects of surface density and size of gold nanoparticles in a fiber-optic localized surface plasmon resonance sensor and its application to peptide detection," Measurement Science and Technology, vol. 21, no. 8, p. 085805, jul 2010. [Online]. Available: https: //doi.org/10.1088\%2F0957-0233\%2F21\%2F8\%2F085805 


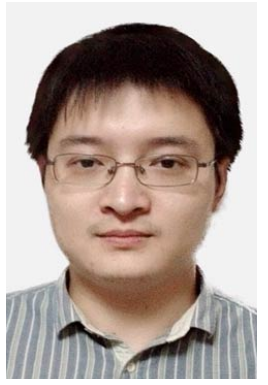

Suoda Chu received the B.Eng. degree (Communication Engineering) from Nanjing University Jinling College, Nanjing, China, in 2010, the MSc degree (Microwave and wireless Communication Engineering) from Cardiff University, Cardiff, UK, in 2014 and is currently working toward his Ph.D degree in school of engineering, University of Aberdeen, Aberdeen, UK.

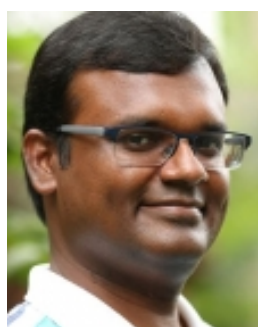

K. Nakkeeran (Ph.D., Fellow IET, CEng, SMIEEE, SMOSA) received the B.Eng. degree from the Coimbatore Institute of Technology, Coimbatore, India, in 1993, and the M.Tech. and Ph.D. degrees from Anna University, Chennai, India, in 1995 and 1998, respectively. In 1999, he joined the Institute of Mathematical Sciences, Chennai, where he was a Post-Doctoral Fellow for ten months. In 1999, he became a Research Associate with the Department of Physics, University of Burgundy, Dijon, France. In 2002, he became a Post-Doctoral Fellow with the Department of Electronic and Information Engineering, The Hong Kong Polytechnic University. In 2005, he joined the School of Engineering, University of Aberdeen, Aberdeen, U.K., where he has been a Senior Lecturer since 2011 His research interests include solitons, fiber lasers, fiber sensors, modeling and simulations of optical devices, long-haul optical fiber communications, and nonlinear science.

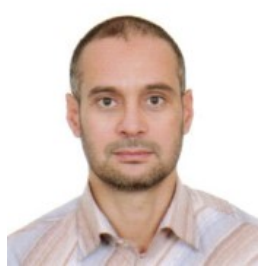

Abdosllam M. Aboboaker received the High Diploma in Electronic Engineering- Higher Institute of Electricity, Zliten, Libya. (September 1995), M.Eng. in Engineering (communication and computer)- National University of Malaysia (UKM) Bangi Selangor, Malaysia.(November 2003), and $\mathrm{Ph} . \mathrm{D}$. in Engineering University of Aberdeen, $\mathrm{Ab}-$ erdeen, UK (November 2009), he joined the College Of Electronic Technology Bani Walid Libya as faculty member in 2010, he became a dean of the college in 2011, he appointed as President of the Constituent Committee for the establishment of the Supreme Council of Technical and Vocational Education, Ministry of higher Education Libya in May 2012, His research interests include solitons, fiber lasers, long-haul optical fiber communications, and nonlinear science modeling and simulations of optical devices,. Dr. Abdosllam is a member of the Optical Society of America and The Institute of Electrical and Electronics Engineers (IEEE).

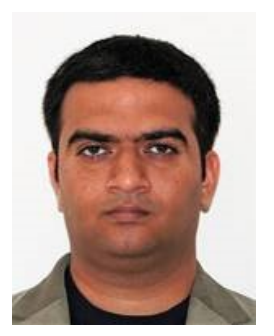

Sumeet S. Aphale (Ph.D., CEng, SMIEEE, MIET) is a Senior Lecturer at the School of Engineering, University of Aberdeen. His has earned the B.E. (University of Pune, India 1999), M.S. (University of Wyoming, USA 2003) and Ph.D. (University of Wyoming, USA, 2005) degrees, all in Electrical Engineering with a focus on Robotics and Control. He has held post-doctoral research positions at the ARC Centre of Excellence for Complex Dynamic Systems and Control, University of Newcastle (2006-08) and at the Centre for Applied Dynamics Research, University of Aberdeen (2008-09). His research interests include nanopositioning, drill-string dynamics, vibration control, flexible manipulators, Gough-Stewart platforms, biomedical devices and fibre-optic sensors; and has published over 60 papers in peer-reviewed journals and conferences. He is an Associate Editor for the IEEE Control Systems Societys Conference Editorial Board. He is also serves as an Associate Editor for Shock and Vibration as well as for Frontiers of Mechanical Engineering (Mechatronics Section).

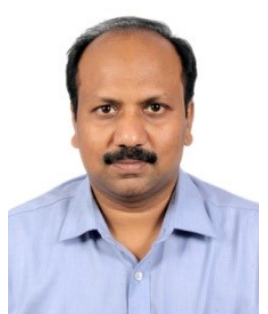

S. Sivabalan received his B. Tech. degree in Control \& Instrumentation Engineering, June 2000, M.Tech. degree in Laser and electro-optical engineering from the College of Engineering, Anna University, Chennai, India in Dec. 2002, and the Ph.D. degree from VIT University, Vellore, India, in July 2013. From 2013 to 2016, he was an Associate Professor at the Department of Electrical Engineering, Vellore Institute of Technology. Since 2017, he has been holding the position of Professor in the School of Electrical Engineering. He was a visiting researcher of Bilkent University, Ankara, Turkey. His research interests include ultrafast fiber laser, fiber optic sensor, microstructured fibers, visible light communication and nonlinear optical imaging.

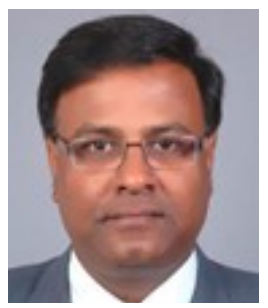

P. Ramesh Babu is a Professor at VIT, Vellore, India. He earned M.Sc., M.Phil., and Ph.D. degrees in Physics from University of Madras, Chennai. His areas of research include fiber Bragg gratings, optical fiber communications, photonic crystal fibers, pulse compression, metamaterials and Bose-Einstein condensation.

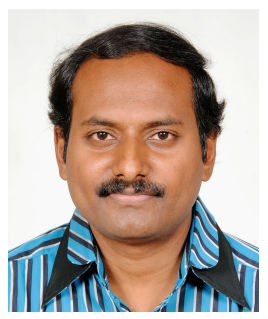

K. Senthilnathan is a Professor at VIT, Vellore. He earned M.Sc and M.Phil. degrees in Physics from University of Madras, and Ph.D. degree from Anna University, Chennai. His areas of research include optical fibers, fiber Bragg gratings, photonic crystal fibers, pulse compression, Bose-Einstein condensation and metamaterials. 